\title{
AN ALTERNATIVE TO THE CAMP SECOND MESSENGER CONCEPT
}

\author{
R. SCHWYZER \\ Institut für Molekularbiologie und Biophysik, \\ Eidgenössische Technische Hochschule Zürich, \\ CH-8049 Zürich, Switzerland
}

\begin{abstract}
A critique of the cAMP second messenger concept is offered in the light of recent experiments with ACTH derivatives, phenoxazones, and rat isolated fat and adrenal cortex cells. It is proposed that enhancement of cAMP production is an event paralleling lipolysis and steroidogenesis, and that it contains an element of 'emergency'. The latter responses might depend on hormonal stimulation of a system capable of utilizing background amounts of cAMP and of producing cAMP homoiostasis. It is experimentally demonstrated for the first time that the fat cell membrane contains dormant ACTH discriminators constituting a discriminator reserve capable of being mobilized and converted to additional hormone receptive, functional discriminators. The findings are discussed in terms of classical receptor theory and of the non-stoichiometric, floating receptor concept.
\end{abstract}

\section{ABBREVIATIONS}

Amino acids and peptides according to the IUPAC-IUB recommendations. ACTH denotes adrenocorticotropic hormone. cAMP, adenosine 3',5'-cyclophosphate. EGTA, ethyleneglycol bis-(dicarboxymethylaminoethyl)-ether.

\section{INTRODUCTION}

I am convinced that the molecular biology of polypeptide hormones is not only a fascinating area because of the interdisciplinary approach necessarily involved in its study, but also an important one, because it promises insight into a poorly understood, but essential function of the cell membrane: information transduction. One- and three-dimensional organization of information in polypeptides and its read-out by macromolecular transducing devices of the target cells, the receptors, have been recurring themes of a number of publications and lectures during the past ten years (References: cf. ref. 1). 


\section{THE CAMP SECOND MESSENGER THEORY}

According to Sutherland and his school, many polypeptide and catecholamine hormones react as first messengers with target cell outer membrane surface 'receptors' to enhance cAMP production by the enzyme adenyl cyclase which is located on the inner membrane surface. The additional, internal cAMP then acts as second messenger, triggering responses which are absolutely cell specific. The theory postulates discrete, causal steps: Hormone $\rightarrow$ cAMP $\rightarrow$ Response, formally resembling the genetic 'dogma': DNA $\rightarrow$ RNA $\rightarrow$ Protein. For example, ACTH, glucagon, and adrenalin induce cAMP production in fat cells (adipocytes, lipocytes), the cell-specific response to these hormones is lipolysis. Lipolysis is supposed to be a consequence of the cAMP concentration rise above background and to be completely detached from any further hormone action. Adrenal cortex cells are believed to respond to ACTH-induced CAMP with enhanced steroidogenesis, melanocytes with melanin granule dispersion. Many hormone actions can be mimicked by external application of mono- and dibutyryl cAMP to target cells. In contrast to cAMP which only in a few cases can replace the hormone, these more lipophilic compounds are believed to penetrate the cell membrane and to be hydrolysed to cAMP in the cytoplasm. An impressive amount of evidence has been accumulated in favour of the cAMP second messenger concept ${ }^{2,3}$.

It has been demonstrated that, in the case of adipocytes, the different hormones react with different, hormone-specific surface 'receptors' or 'discriminators'4-7. The hormone-'receptor' complexes then react with one and the same set of adenyl cyclase enzyme molecules, triggering their action. A careful kinetic analysis of published data from well-documented sources ${ }^{8}$ supports this view and stresses the points that: (i) there need not be a stoichiometric relationship between hormone 'receptors' and adenyl cyclase, (ii) that the affinity between 'receptors' and adenyl cyclase is greatly increased when hormone is 'bound' to the 'receptor', and (iii) that the 'receptor' and/or the adenyl cyclase molecules can be freely 'floating' in the membrane (lateral diffusion). The author calls this the "non-stoichiometric floating receptor model'.

In the following. I shall call the hormone-specific, functional binding sites on the outer surface of the target cell plasma membrane discriminators (according to refs. 9, 10), the enzymes or other proteins on the inside which are directly stimulated by the discriminator-hormone complex apo-receptors, and the discriminator-apo-receptor system (complex) holo-receptor.

\section{QUESTIONING THE cAMP SECOND MESSENGER CONCEPT}

Lang and Schwyzer ${ }^{11}$ have recorded a case that is difficult to explain by the second messenger concept. We found that $3.5 \times 10^{-8} \mathrm{M}$ ACTH produces in isolated lipocytes both a short burst of cAMP that reaches its maximum within five minutes after hormone application and decreases to background levels within about twenty minutes, and a prolonged (1.5-2 hours), steady, increased rate of lipolysis. The two phenoxazones tested, actinocin (2-amino4,6-dimethyl-phenoxazone-(3)-1,9-dicarboxylic acid) and its peptido deriva- 
tive, actinomycin D, almost double the rate of ACTH-induced lipolysis, but decrease the accumulation of cAMP to zero (no increase above background levels). This could mean that (i) ACTH-sensitive lipolytic holo-receptors that can be made more responsive by phenoxazones occur in parallel to the adenyl cyclase holo-receptors which are desensitized by phenoxazones, or that (ii) ACTH-specific holo-receptors exist which simultaneously utilize background amounts of cAMP to produce lipolysis and operate a regulation mechanism for cAMP homoiostasis. Activation of adenyl cyclase and negative feedback would be essential for cAMP regulation according to (ii). Ho and Sutherland ${ }^{12}$ have postulated formation and release of a hormone antagonist by cAMP in adipocytes; its relevance in connection with our findings remains to be investigated.

Our subsequent work in this area has substantiated our doubts as to the validity of the cAMP second messenger concept, has revealed an unexpected phenomenon: 'Discriminator Reserve Mobilization', and has given support to the concept of non-stoichiometric floating discriminators and aporeceptors.

\section{DISCRIMINATOR RESERVE MOBILIZATION}

In order to complement our knowledge on hormone receptor interactions from a different viewpoint, we decided to investigate the number of ACTH binding sites on isolated adipocytes, some thermodynamic properties of the hormone binding site complex in situ, and the possible influence of phenoxazones and EGTA (calcium depletion and ACTH action inhibition ${ }^{13-16}$. Our results have, in part, been preliminarily communicated ${ }^{1,17}$.

We synthesized chemically (from amino acids) the ACTH analogue [2-phenylalanine, 4-(4',5'-dehydro-4', $5^{\prime}$-ditritio)-norvaline]-ACTH-(1-24)tetrakosipeptide, $\left(\mathrm{Phe}^{2}, \mathrm{Nva}\left(\mathrm{t}_{2}\right)^{4}\right)-\mathrm{ACTH}-(1-24)$ in a very pure state. It was found to be a full agonist with regard to lipolysis in rat isolated lipocytes, and to steroidogenesis and cAMP production in rat isolated adrenal cortex cells. However, its potency is about ten times lower than that of the corresponding peptide with the natural sequence, ACTH-(1-24), containing tyrosine and methionine in positions 2 and 4 , respectively ${ }^{18,19}$. We used the compound for binding studies with isolated adipocytes, prepared according to Rodbell ${ }^{20}$. Specific, reversible binding was measured by the method of Cuatrecasas ${ }^{21}$, adapted to our problem.

The principal results from some 100 experiments are:

(i) The isolated adipocytes are a nearly Gaussian-distributed population of spherical cells with diameters ranging from 20 to $100 \mu$. The mean diameter was found to be $49.4 \pm 7.0 \mu$ with 95 per cent confidence limits of $\pm 0.18 \mu$. The mean surface area is $62.4 \times 10^{6} \mu^{2}$ per 7800 cells $(1 \mathrm{mg}$ dry weight). These results were obtained with a Coulter Counter, courtesy of Dr Peter Moser, Basel.

(ii) In the concentration range of $5 \times 10^{-10}$ to $5 \times 10^{-8} \mathrm{M}$ hormone analogue, binding is very rapid and easily reversible (at $37^{\circ} \mathrm{C}$, equilibrium is reached within one to two minutes). 


\section{R. SCHWYZER}

(iii) In the same concentration range, with and without addition of $10^{-6} \mathrm{M}$ phenoxazone, apparently one homogeneous class of binding sites is operative (linear correlation coefficients 0.93 and 0.96 , respectively).

(iv) The apparent association constants are: $K_{\text {ass }}=7.3( \pm 0.8) \times 10^{7} \mathrm{M}^{-1}$ (Scatchard), $9.3 \times 10^{7} \mathrm{M}^{-1}$ (Hill), and $7.8 \times 10^{5} \mathrm{M}^{-1}$ (Lineweaver-Burk) without phenoxazone, and $K_{\mathrm{acs}}=6.7( \pm 1.2) \times 10^{7} \mathrm{M}^{-1}$ (Scatchard). $9.7 \times 10^{7} \mathrm{M}^{-1}$ (Hill), and $8.7 \times 10^{7} \mathrm{M}^{-1}$ (Lineweaver-Burk) with phenoxazone. The Hill coefficient, a certain measure of cooperativity, was found by an iterative method ${ }^{22}$ to be of the order of 1.3 in both cases.

(v) The mean apparent dissociation constant values, $K_{\text {diss }}=1.2( \pm 1) \times$ $10^{-8} \mathrm{M}$ (with and without phenoxazone) coincide very nicely with those calculated from the lipolytic potency (hormone concentration at halfmaximal response), namely $8.7( \pm 3.3) \times 10^{-9} \mathrm{M}$. The potency was recalculated with a non-linear iteration procedure (programme DOLORES) from the data recorded in the paper by Lang et al. ${ }^{19}$ by Dr J.-L. Fauchère in our laboratory.

(vi) The number of binding sites per square micrometre was calculated as: 316 (Scatchard) and 287 (Hill), without, and 582 (Scatchard) and 472 (Hill), with phenoxazones. Phenoxazones cause a mean increase of binding sites of about 175 per cent (530 versus 300 ) which again corresponds closely to the observed increase of lipolysis rates ${ }^{11}$.

(vii) Although $\mathrm{Ca}^{2+}$ depletion by EGTA abolishes the lipolytic response completely, no change of binding properties is observed.

(viii) Assuming that one macromolecular binding site would occupy approximately an (ample) membrane surface area of $2500 \AA^{2}$, we find that 0.75 per cent of the cell surface is occupied by ACTH binding sites without. and 1.3 per cent with added phenoxazones. Lefkowitz, Pastan and Roth ${ }^{23 \ldots 25}$ arrived at the following figure for ACTH binding: 360000 sites per cell $\left(K_{\mathrm{ass}}=3 \times 10^{7} \mathrm{M}^{-1}\right)$, or 600 sites $/ \mu^{2}$, or $1.5 \mathrm{per}$ cent of the cell surface reserved for ACTH binding (recalculated on the assumption that the cells are cubic with $10 \AA$ sides).

(ix) The apparent dissociation.constant for the peptide with the natural sequence, ACTH-(1-24), is approximately $1.2( \pm 3) \times 10^{-9} \mathrm{M}$ (from binding inhibition studies), again comparing well with the apparent lipolytic potency value of $7.5( \pm 2) \times 10^{-10} \mathrm{M}^{19}$.

(x) Other lipolytic hormones, like glucagon and adrenaline, do not inhibit $\left[\mathrm{Phe}^{2}, \quad \mathrm{Nva}\left(\mathrm{t}_{2}\right)^{4}\right]-\mathrm{ACTH}-(1-24)$ binding. They do not interfere with ACTH $-(1-24)$ binding competition. This strongly supports the view mentioned above that different hormones bind to different discriminator sites ${ }^{4-7}$. Antagonists towards ACTH action in isolated adrenal cells, p.e. ACTH-(7-24)octadecapeptide (unpublished) and ACTH-(11-24)-tetradecapeptide ${ }^{26}$, also antagonize fat cell binding.

The conclusions from this part of our study are the following:

(i) The observed binding sites are functional discriminators of holo-receptors functionally connected with lipolysis because of (a) the parallel influences of the phenoxazones on binding site number and on lipolysis rate, (b) the characteristics of binding inhibition by various agonists and antagonists (vide supra), and (c) the coincidence of the apparent dissociation constants 
derived from binding and from lipolysis data (for possible objections to this last point, vide infra).

(ii) The fat cell membranes contain a discriminator reserve that can be mobilized from a dormant to a hormone receptive state of the molecules.

(iii) If discriminator reserve mobilization by the phenoxazones were found to have its physiological correlate (not involving phenoxazones!), this would indicate that hormonal regulation would not only depend on hormone blood levels, but also on the variable receptivity of target cells (proportional to the discriminator number).

\section{HORMONE-GUIDED CAMP ACTION}

\section{Survey}

One of the critical points in the cAMP second messenger theory is the difficulty of explaining the fact that in spite of existing, sometimes rather high background CAMP levels, relatively small concentration rises should be able to produce the hormonal response. To resolve the problem, the idea of cAMP compartmentalization is often invoked. Compartmentalization is visualized as the existence of different CAMP chemical potentials at various strategic points within the cell. Results described in the following paragraphs suggest that the sensitivity of certain mechanisms utilizing cAMP for response generation is increased by the hormone, so that even cAMP background levels become operative.

Should, however, cAMP resources begin to decline, response intensity to decrease, and, consequently, larger amounts of hormone to appear in the blood, then adenyl cyclase would be activated and more cAMP produced. This view agrees with the (new) fact that, at least in isolated adrenal and fat cells, cAMP accumulation operates on a considerably higher hormone level than either lipolysis or steroidogenesis.

Activation of adenyl cyclase can be the result of (i) stimulation by hormone-discriminator complex of the adenyl cyclase apo-receptor, and/or (ii) the attenuation of a feedback mechanism which inhibits adenyl cyclase and/or activates cAMP degradation (phosphodiesterase), or does both, and originates somewhere within the hormone-sensitive step: cAMP $\rightarrow$ Response. Elevation of cAMP concentration by hormone is thus not the stimulus for target cell response, but bears certain characteristics of an energency event. (It should be pointed out that in many studies on cAMP stimulation, hormone doses are far in excess of the physiological range necessary for overt response.)

The revised scheme would then be (assuming feedback mechanisms):

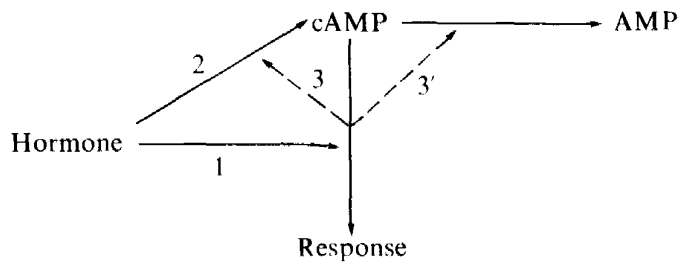


wherein: 1 denotes the hormone's principal action (stimulation of cAMP-toresponse conversion and, more generally, response generation): 2 is the hormone's auxiliary action (adenyl cyclase stimulation): $3,3^{\prime}$ are feedback regulation where 3 is inhibition of cyclase, and $3^{\prime}$, activation of cAMP phosphodiesterase.

\section{Definitions}

A few definitions which might be useful for reading the next paragraphs are as follows:

$[\mathrm{H}] .[\mathrm{D}]$, and $[\mathrm{A}]$ : hormone, discriminator, and apo-receptor concentrations.

$[\mathrm{H}]_{50}$ : hormone concentration necessary for eliciting 50 per cent of a given. maximal response (indicated as superscript).

Oy: occupancy, expressed as observed discriminator occupation by hormone divided by maximal occupation, or hormone discriminator complex concentration divided by the total concentration of receptive discriminator:

$$
\mathrm{Oy}=\mathrm{O} / \mathrm{O}_{\max }=[\mathrm{HD}] /[\mathrm{D}]_{\mathrm{tot}}
$$

Ry: responsivity (fractional response). given by observed response intensity divided by maximal response intensity:

$$
\mathrm{Ry}=\mathrm{R}_{/} \mathrm{R}_{\max }: \quad \mathrm{R}=\mathrm{R}_{\max } \therefore \mathrm{Ry}=1 \text { ( or } 100 \text { per cent) }
$$

\section{ACTH potency differences between CAMP and response stimulation}

Comparison of $\log$ dose/response curves for the stimulation of steroidogenesis and CAMP accumulation in isolated adrenal cortex cells by ACTH and various analogues ${ }^{27.28}$ reveals one striking characteristic: the $[\mathrm{H}]_{50}$ value for cAMP is almost invariably 20 to 40 times greater than that for steroidogenesis. Observations on isolated fat cells reveal the same general trend with probably an even greater difference (unpublished). The situation is schematically illustrated in Figure 1. According to the cAMP second messenger theory this would imply that a $0-2$ per cent increase of cAMP over background levels would suffice to cause $15-30$ per cent totally possible steroidogenesis. Classical receptor theory would explain this with the assumption of a large receptor reserve (cf. Rudinger, Pliška and Krejči ${ }^{29}$ ). However, a device sensitive enough to work with such small concentration differences against a large, fluctuating background is hard to imagine. Furthermore, our binding studies have introduced a new complication, because the binding curves coincide with lipolysis and not with CAMP or cyclase stimulation curves. We envisage two possible explanations based on classical receptor theory.

The first one, shown in Figure 2, assumes c AMP to be the second messenger. It combines two features observed in isolated adrenal and fat eells into an idealized cell.

(i) Responsivity for response 1 (R1) requires a 20 times larger hormone concentration than equal responsivity for response $2(\mathrm{R} 2)$, if response 1 is 


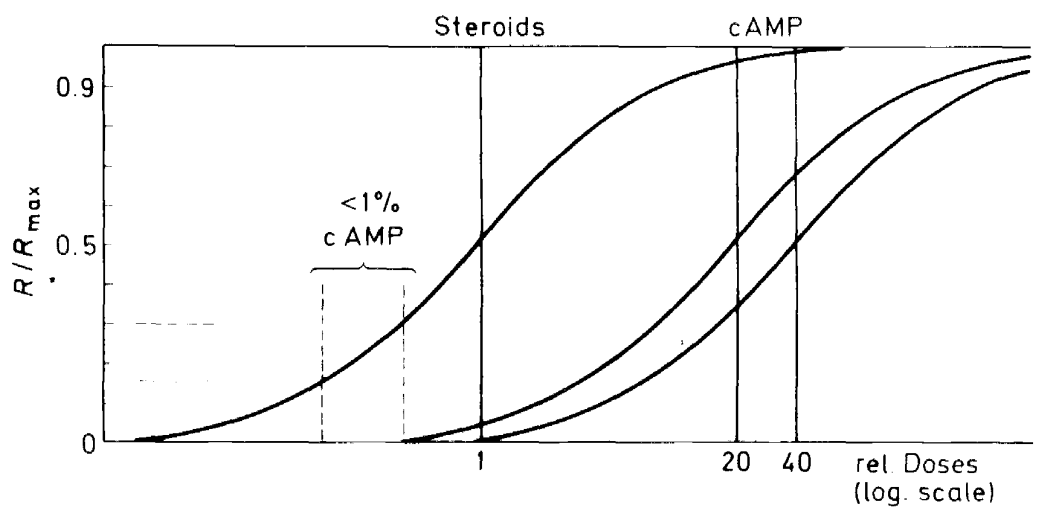

Figure 1. Relative log dose/response relationship for ACTH agonists in isolated adrenal cells

identified with cAMP accumulation and response 2 with steroidogenesis or lipolysis:

$$
\mathrm{Ry}^{1}=\mathrm{Ry}^{2} \therefore[\mathrm{H}]_{50}^{1} /[\mathrm{H}]_{50}^{2}=20
$$

(ii) The ratio $[\mathrm{H}]_{50}^{0} /[\mathrm{H}]_{50}^{2}=1$. This reflects the observed coincidence between occupancy (binding) and lipolysis curves in isolated lipocytes. If cAMP is regarded as the second messenger, this coincidence must be purely fortuitous, and the information transduction events the following: (a) Occupancy of discriminators produces a proportional stimulus which activates the apo-receptor adenyl cyclase. In order to shift the $\mathrm{R} 1$ versus $\log [\mathrm{H}]$ curve to 20 times larger hormone concentrations, a large discriminator or
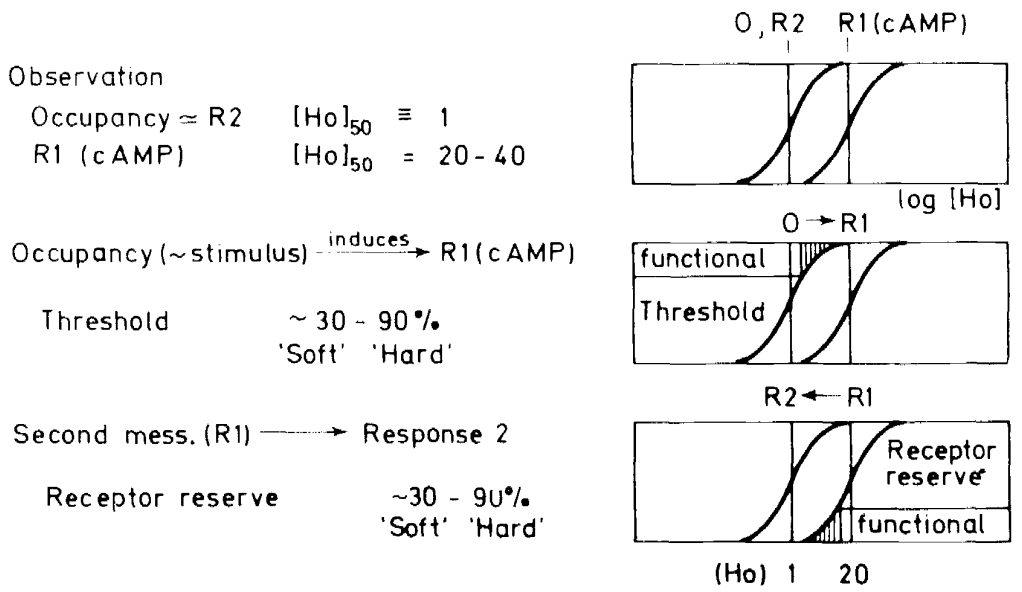

Figure 2. Receptor events assuming $\mathrm{cAMP} \equiv$ second messenger (occupancy $\simeq \mathrm{R} 2$ is fortuitous coincidence) 


\section{R. SCHWYZER}

stimulus threshold must be overcome, before cAMP production substantially starts. This threshold amounts to 90 per cent of the discriminators (or of their stimuli) in the case of a linear occupancy-to-R1 relationship ('hard' threshold). If a non-linear relationship is operative, the threshold must be 'soft' and become increasingly 'leaky' above about 30 per cent occupancy. (b) In a second step, cAMP is converted to response 2 in such a manner that the $\mathrm{R} 2$ curve is shifted back to 20 times lower $[\mathrm{H}]_{50}$. According to classical theory, this can be accomplished with a large receptor reserve. In our case, only between $0-10$ per cent or $0-30$ per cent of the cAMP that can be produced maximally is actually needed for eliciting a full response $2(\mathrm{R} 2=1)$, assuming 'hard' or 'soft' receptor reserves, respectively.

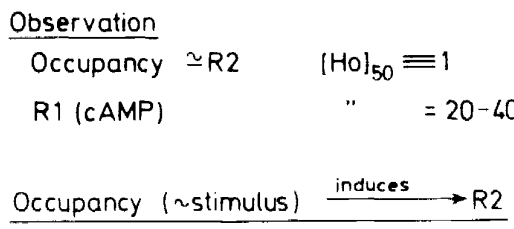

No observable threshold.

Slight receptor reserve?

Occupancy ( ustimulus') $\longrightarrow R 1$ (cAMP)

Parallel event with high threshold.

cAMP production only in emergency (high

hormone concentrations)?

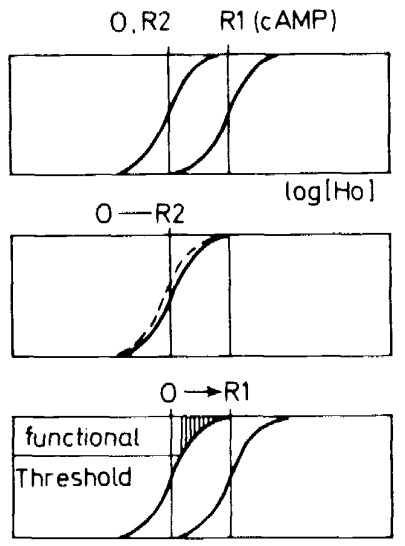

Figure 3. Receptor events assuming cAMP $\neq$ second messenger (occupancy $=\mathrm{R} 2$ is meaningful coincidence)

The second explanation, based on classical receptor theory is shown in Figure 3. It assumes cAMP not to be the second messenger for $\mathrm{R} 2$. The suppositions and idealized observations are the same as in Figure 2. The coincidence between occupancy and $\mathrm{R} 2$ now becomes more meaningful in the sense that there is a closer connection between the two. The stimulus initiated by discriminator occupancy would more directly induce R2, and neither threshold nor receptor reserve need be invoked (in the reality of the isolated adipocyte, a small difference between $K_{\text {diss }}$ for occupancy and R2 might call for a slight receptor reserve). The same occupancy, or another occupancy $\left(\mathrm{O}^{\prime}\right.$, not separately observable because of too small a difference in $K_{\text {ass }}$, involving either the same or another set of discriminators, vide infra) and its derived stimulus ( $\left.\mathrm{S}^{\prime}\right)$, would then induce $\mathrm{R} 1$. This would be an event parallel to that of R2 induction. Its purpose would be different (emergency? homoiostasis of background cAMP?), it would require higher hormone concentrations than $\mathrm{R} 2$, and be operative with a high apparent $\mathrm{O} \rightarrow \mathrm{R} 1$ threshold.

Quite obviously, the second mechanism is simpler and more straightforward. Keeping in mind the inadequacies of our assumptions and generali- 
zations, the preliminary nature of many observations, and the fact that they are derived from cells in isolated state instead of in situ, the conclusions must remain only suggestive until more material accumulates in their favour. Such material will be discussed in the following paragraphs.

\section{Dissociation of cAMP from response stimulation}

$\left(\mathrm{N}^{\varepsilon}\right.$-Dansyllysine $\left.{ }^{21}\right)-\mathrm{ACTH}-(1-24)$-tetrakosipeptide ${ }^{30-33}$ is a full agonist [same maximal response as ACTH-(1-24)] in isolated adipocytes and adrenal cortex cells with respect to lipolysis and steroidogenesis (R2), and cAMP (R1, measured as adenyl cyclase activation in fat cell ghosts and as cAMP accumulation in isolated adrenal cells $\left.{ }^{27}\right)$. Whereas its potency is between five and twenty times lower $\left([\mathrm{H}]_{50} \simeq 5-20\right.$ times higher) than that of ACTH (1-24) in the R2 experiments, its potency with respect to cAMP (R1) is equal. Here, we have an exceptional case with $[\mathrm{H}]_{50}^{1} /[\mathrm{H}]_{50}^{2}=0.2-0.05$ instead of 20-40. Within a certain hormone concentration range, a large amount of cAMP will be stimulated, but only a very small amount of lipolysis or steroidogenesis. This is indicative of a drastic change in the threshold and receptor reserve situation induced by the dansyl substitution. The dansyl group lies well to the far side of the address part ${ }^{1}$ of the molecule, but it could influence $\operatorname{Trp}^{9}$, which is an essential component of the message part, by electronic interference ${ }^{33}$.

In the light of the 'non-stoichiometric floating discriminator/apo-receptor model', we could envisage a differential interaction between the ACTHanalogue:discriminator complex and two apo-receptors:adenyl cyclase on the one hand, and a lipolytic or steroidogenic apo-receptor on the other:

$$
\begin{array}{ll}
\{\mathrm{HD}\}+\mathrm{A}^{1} \rightleftharpoons\left\{\mathrm{HDA}^{1}\right\} & K_{\mathrm{A}}^{1} \\
\{\mathrm{HD}\}+\mathrm{A}^{2} \rightleftharpoons\left\{\mathrm{HDA}^{2}\right\} & K_{\mathrm{A}}^{2} \\
\left\{\mathrm{H}^{\prime} \mathrm{D}\right\}+\mathrm{A}^{1} \rightleftharpoons\left\{\mathrm{H}^{\prime} \mathrm{DA}^{1}\right\} & K_{\mathrm{A}}^{1} \\
\left\{\mathrm{H}^{\prime} \mathrm{D}\right\}+\mathrm{A}^{2} \rightleftharpoons\left\{\mathrm{H}^{\prime} \mathrm{DA}^{2}\right\} & K_{\mathrm{A}}^{2}
\end{array}
$$

In these apo-receptor-holo-receptor equilibria (subscript $\mathrm{A}$ of the apparent association constants $K), \mathrm{H}$ is reference $\mathrm{ACTH}-(1-24), \mathrm{H}^{\prime}$ is [Lys(Dns) ${ }^{21}$ ACTH-(1-24), D is the (common) discriminator, $\mathrm{A}^{1}$ is the cyclase, and $\mathrm{A}^{2}$ the R2 apo-receptor. Our situation can be explained in terms of differences between the respective $K$-values:

$$
{ }^{\prime} K_{\mathrm{A}}^{1}=K_{\mathrm{A}}^{1} ; \quad K_{\mathrm{A}}^{2}<K_{\mathrm{A}}^{2}
$$

This is identical with saying that the stimulus produced by hormone derivative binding is different from that produced by reference hormone binding (vide infra).

The theme recurs in some recent observations on the behaviour of isolated adrenal cells in the presence of some new, synthetic ACTH-analogues ${ }^{28}$. The differential stimulation of steroidogenesis (R2) and cAMP accumulation (R1) is dependent on details in the message part (amino acid residues $\sim 5-10$ ) of the hormone. ACTH-(7-23)-amide is a competitive inhibitor for both R1 and R2. The analogue ACTH-(7-24) was found by Ursula Lang and Jean-Luc Fauchère in my laboratory to behave similarly with adipocytes. Both contain the strongly reduced message sequence (7)Phe-Arg-Trp-Gly- 


\section{R. SCHWYZER}

attached to the address sequence 11-23 or 11-24. N-terminal addition of histidine No. 6 produces ACTH-(6-24)-nonadecapeptide, a partial agonist for $R 2$ [reaching 40 per cent of the maximal response attainable by ACTH(1-24)], but an antagonist to $\mathrm{Rl}$. ACTH-(5-24)-eikosapeptide is a full agonist for $\mathrm{R} 2$ and a 45 per cent partial agonist for $\mathrm{R} 1$, with a ratio $[\mathrm{H}]_{50}^{1}[\mathrm{H}]_{50}^{2}=31$. Elongation of the hormonal message from residue $7 \rightarrow 5$ produces first a stimulation of R2 and second a stimulation of 'second messenger' which is the inverse sequence to that postulated by the second messenger theory. Steroidogenesis can be triggered in isolated adrenal cells despite antagonistic blockade of adenyl cyclase.

A strong dissociation is observed for $\left(\mathrm{N}^{\text {Ind }}\right.$-nitrophenylsulphenyl-tryptophane ${ }^{9}$ - ACTH-1-39)-nonatriakontapeptide: it is a 77 per cent agonist for R2 and a 99 per cent antagonist with only very weak ( 1 per cent) agonistic properties for R1. [H $]_{50}^{2}$ is 100 times that of ACTH $-(1-24)^{28}$ : Ramachandran reports similar results ${ }^{34}$.

In conclusion, then, these results show that with chemical manipulation of the information in the hormonal message part alone. stimulation of the cAMP response can be dissociated from that of steroidogenesis and lipolysis. They call for the consideration of two receptors, one for adenyl cyclase, and one for R2 stimulation. A preliminary explanation was offered on the basis of the 'non-stoichiometric floating discriminator/apo-receptor model'.

Further experiments, summarized below, indicate that, apart from having two receptor mechanisms [ 1 and 2 in scheme (1)], we are most probably also dealing with feedback mechanisms [scheme(1)], and with a situation involving one type of discriminator and (at least) two types of apo-receptor [scheme (2)].

\section{Isolation of the ACTH mechanism}

We found that. in the adipocyte (the adrenal cortex cell is presently being investigated), the hormonal mechanism of ACTH can be distinguished from that of other hormones. Phenoxazones and EGTA ( $\mathrm{Ca}^{2+}$-depletion) are the tools used in our study.

After careful control without hormone, with other hormones, with dibutyryl cAMP, and with different antibiotics, the results summarized in Figure 4 were obtained with whole isolated adipocytes in the presence of ACTH (adrenaline and glucagon behave quite differently):

(i) Standard events: ACTH-(1-24) (natural amino acid sequence) is bound by approximately 300 discriminators per $\mu^{2}$ cell surface with $K_{\text {ac }}=10^{9} \mathrm{M}^{-1}$ : this is assumed to be the normal, or standard discriminator situation $(+)$. This quantity and quality of binding is expected to give rise to a stimulus (signal) of normal strength (indicated by the simple arrow, $\rightarrow$ ) directed towards the cyclase apo-receptor. The response of the cyclase is indicated in terms of cAMP accumulation above background values (about 150-250 per cent of background) versus time. The peak is reached after about five minutes following the application of $3.5 \times 10^{-8} \mathrm{M} \mathrm{ACTH-(1-24).} \mathrm{This} \mathrm{is}$ assumed to be the standard response, and the integrated amount of cAMP present above background is designated as $(+)$. The stimulus resulting from this amount of 'second messenger' is, again, taken as standard $(\rightarrow)$; so is the observed lipolysis rate enhancement $(+)$. 


\begin{tabular}{|c|c|c|c|c|c|c|c|}
\hline & & DISCR. & & CYCLASE & 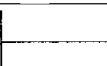 & LIPOLYSIS & \\
\hline$\overline{k_{\text {ass }}}$ & $D / \mu^{2}$ & $0=10^{-9} \mathrm{t}$ & $\left.10^{-7} \mathrm{M}\right)$ & & & & ADIPOCYTES \\
\hline $10^{9}$ & 300 & + & $\rightarrow$ & $n+$ & $\longrightarrow$ & + & ACTH $\quad\left(-35 \times 10^{-8} \mathrm{M}\right)$ \\
\hline $10^{9}$ & 300 & + & $\longrightarrow$ & $\angle++$ & $\Rightarrow$ & $\square$ & + EGTA $\times 10^{-3}\left(-\mathrm{Ca}^{2+}\right)$ \\
\hline $10^{9}$ & 600 & ++ & $\Rightarrow / 1$ & $L^{-}$ & $\rightarrow \rightarrow$ & $+=++$ & $+{ }^{\prime}$ PHENOXAZONE' $\times 10^{-6}$ \\
\hline $10^{9}$ & 600 & ++ & $\Rightarrow$ & $\simeq++$ & $\Rightarrow / /$ & & +EGTA + PHENOX. \\
\hline & & & & & & & 'GHOSTS' \\
\hline & & $(+)$ ? & & + & & & ACTH $\quad\left(-5 \times 10^{-8} M\right)$ \\
\hline & & $(+) ?$ & & $-+(-)$ & & & + EGTA $\left(-\mathrm{Ca}^{2+}\right)$ \\
\hline & & $(++) ?$ & & ++ & & & +PHENOXAZONE \\
\hline & & $(++) ?$ & & $\sim++(-)$ & & & +EGTA + PHENOX \\
\hline
\end{tabular}

Figure 4 .

(ii) Pre-incubation (ten minutes) with $10^{-3} \mathrm{M} E G T A$ before hormone addition (reducing considerably the $\mathrm{Ca}^{2+}$ levels, cf. refs. 13-16) causes no change at the discriminator level $(+)$ and, presumably, of the stimulus $(\rightarrow)$, but cAMP production appears sustained, and its accumulation is considerably higher $(++)$ than without EGTA. We would therefore expect the "second messenger stimulus' to be enhanced $(\Rightarrow)$ and to call for an increased rate of lipolysis over that obtained with hormone alone. However, EGTA produces a block ( ) in the cAMP-to-lipolysis coupling mechanism, and no lipolysis is observed $(-)$.

(iii) Pre-incubation with, or even mere simultaneous addition together with the hormone of the two phenoxazones tested (actinocine and actinomycin D) leaves $K_{\text {ass }}$ unaltered, but approximately doubles the amount of discriminators per target cell. Hormone quantities bound are also doubled $(++)$, and we would expect a stronger total stimulus to ensue from the greater number of hormone-discriminator complexes $(\Rightarrow)$. But, apparently, stimulus-cyclase coupling is blocked (), as no cAMP accumulation above background can be observed (-). Although we would expect the 'cAMP stimulus' towards lipolysis to be extremely weak $(\rightarrow)$, a strongly enhanced lipolysis rate (about 180 per cent) above that achieved with ACTH alone is the result $(++)$. If additional cAMP is needed for lipolysis, then its weak stimulus must, in this case. be very considerably magnified $(<)$. We would have to postulate a very sensitive device that can sense and make use of the slightest increases of cAMP concentration (order of magnitude of one per cent, vide infra) over a strong background.

(iv) Pre-incubation with a combination of $10^{-3} \mathrm{M}$ EGTA and $10^{-6} \mathrm{M}$ phenoxazone enhances both discriminator number $(++)$ and cAMP accumulation $(++)$. Thus, EGTA abolishes the apparent block between discriminator and apo-receptor observed for phenoxazone alone. However, the EGTA block between cyclase and lipolysis (-) still persists, despite the 


\section{R. SCHWYZER}

presence of phenoxazone which would, per se, have been expected strongly to enhance lipolysis -especially in the presence of increased amounts of cAMP.

In order to supplement our information at a reduced level of system complexity. similar experiments were carried out with fat cell 'ghosts', which consist mainly of plasma membranes ${ }^{35}$. The ghost discriminator binding studies still remain to be performed: therefore. the anticipated results shown in Figure 4 bear a question mark. Lipolysis, which is peculiar to the cytoplasm, is detached, and only the 'isolated' response of the membrane adenyl cyclase was measured.

(v) $5 \times 10^{-8} \mathrm{M}$ ACTH-(1-24) produced the standard increase of ATP cAMP conversion rate $(+)$.

(vi) Contrary to what could have been expected from the behaviour of whole lipocytes (ii), EGTA reduced adenyl cyclase activity to about a half to one quarter of the standard $(+-)$. This agrees with the results of others ${ }^{13} \ldots$ and suggests that the observed accumulation of CAMP in whole cells has something to do with the unimpaired connection of plasma membrane with the cytoplasm.

(vii) Also contrary to our expectation from the behaviour of whole cells (iii), the phenoxazones don't decrease cyclase activity, but almost double it, which parallels the discriminator reserve mobilization observed in the intact isolated cells. The phenoxazone block of cAMP accumulation (iii) isn't located in the cell membrane (discriminator stimulus apo-receptor coupling). but is intimately connected with the presence of the cytoplasm. If as we presume, discriminator reserve mobilization can also occur in the fat cell ghost then the plasma membrane would be an essential point of phenoxazone action.

(viii) Combined application of EGTA and phenoxazones activates adenyl cyclase to about 150 per cent above the level observed with hormone alone $(++-)$. In the light of vi and vii. this behaviour was expected.

\section{A working hypothesis for hormone-guided CAMP action}

I have attempted to interpret all these findings in terms of a working hypothesis. illustrated in Figure 5 . which is a more detailed version of scheme (1) at the beginning of this paper. Hopefully, the questions raised by this hypothesis will help us plan and execute further relevant experiments. The various boxes represent regulatory and other functional "elements, and should not necessarily be identified with protein molecules. The double line separates their locations into those in or on the fat cell plasma membrane ("ghost") and those more inside the cell ("cell").

Discriminator reserve mobilization is indicated at the left. The (reversible?) transition from dormant (off) to hormone receptive (on) is depicted as a conformational change affecting recognition site topography ${ }^{36}$. Phenoxazones appear to act on the cell membrane tenhancement of ghost cyclase stimulation). I would like to call the hypothetical, functional entity on which they act Moderator of ACTH Stimulus, and would again stress the point that this is not necessarily an apo-receptor or other macromolecule, but is a designation for a complex function, a function that in reality might depend 
on the interaction of many molecules and ionic species of the cell membrane. Phenoxazone action on the 'Moderator' effects (i) holo-receptor mobilization [via discriminator reserve mobilization and perhaps, changes of $K_{\mathrm{A}}^{1}$ and $K_{\mathrm{A}}^{2}$ (2)]. (ii) lipolysis enhancement, and (iii) reduction of cAMP accumulation.

The last two effects are only operative in the whole cell (lipolysis and cAMP reduction are missing in the ghost). I assume another hypothetical, functional entity, the ACTH-sensitive Transducer of cAMP Information, located directly on or near the inside surface of the plasma membrane. Its

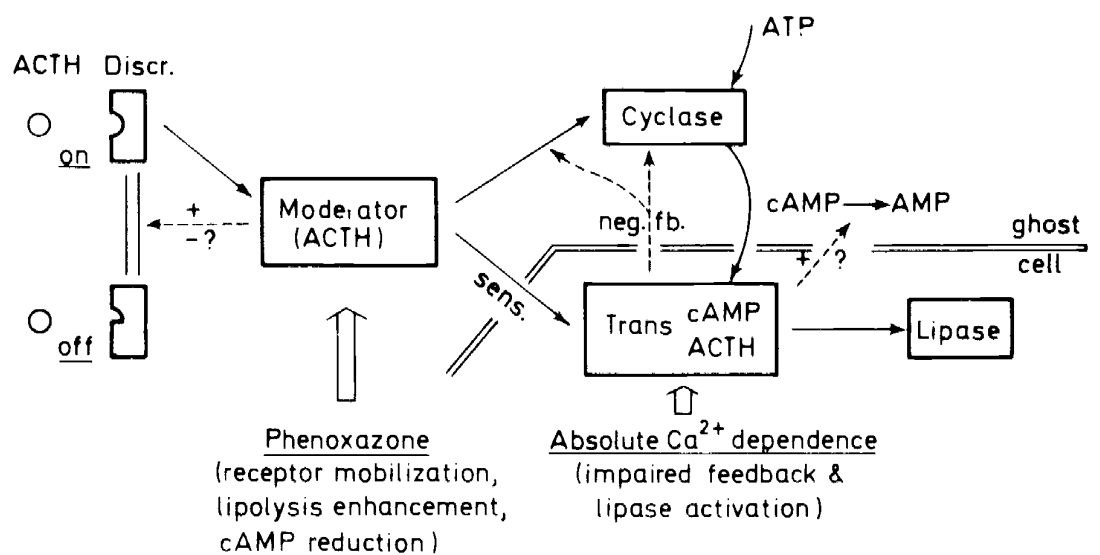

Figure 5.

functions are (i) transformation of cAMP information into lipolysis (via kinase activation?), (ii) exertion of a negative feedback on adenyl cyclase activation (effect direct or via lowering of $K_{\mathrm{A}}^{1}(\mathbf{1})(\mathbf{2})$ ?), and (iii) possibly (?) an activation of phosphodiesterase. The 'Transducer' is stimulated via 'Moderator' and sensibilized to the pre-existing, background levels of CAMP. This could be accomplished p.e. by the enhancement of the $K_{\text {ass }}$ of an essential component for cAMP which would be equivalent to a change in compartmentalization. At the same time, the 'Transducer', which is also a 'Sensor' for cAMP concentration, exercises a negative feedback which persists as long as a sufficient supply of cAMP is present (if the target cell is challenged with an unphysiologically large supply of hormone, cAMP will 'overshoot' before becoming subject to the feedback, cf. the curves in ref. 11.) Phenoxazones amplify the hormone stimulus via 'Moderator', enhancing (i) reduction of cAMP accumulation in whole cells and (ii) adenyl cyclase stimulation in the ghost (feedback missing because 'Transducer' is missing).

The 'Transducer' is inhibited by EGTA (needs $\mathrm{Ca}^{2+}$ for normal function?) which is indicated by the EGTA-produced impairment of (i) lipase activation and (ii) reduction of cAMP accumulation in the whole cell (ghost adenyl cyclase is only weakly affected by EGTA and, if anything, its activity is 


\section{R. SCHWYZER}

reduced, which would not explain the enhanced, sustained cAMP levels in the whole cell). The sustained cAMP levels in the whole cell are best explained by a lack of an originally present feedback mechanism.

Figure 5 can be simplified to the following scheme (3) which avoids involuntary identification of hypothetical functional entities with molecules:

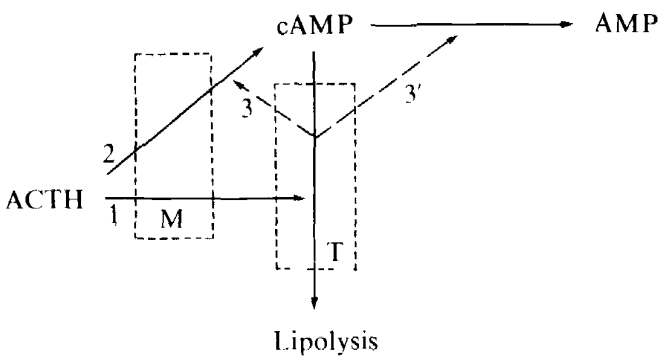

wherein: $M$ signifies moderation of ACTH stimulus and discriminator reserve mobilization (point of phenoxazone action); $T$ is transduction of cAMP into lipolysis activation and feedback (point of EGTA action); other numbers, see scheme (1).

\section{A HYPOTHETICAL MOLECULAR RECEPTOR MODEL}

In concluding, I would like to present the molecular model that is the result of the foregoing discussions and represents a working hypothesis upon which our future experiments will be based (Figure 6). The polypeptide hormone can be either flexible or have a more or less fixed conformation ${ }^{36}$. Examples for both types are known ${ }^{1}$. Those conformers which have a fitting 'message' and 'address' topography will be specifically bound to a complementary topographic unit exposed on the 'receptive' discriminators. Receptive discriminators can be mobilized from a 'dormant' reserve (either wrong topography or submerged). The hormone-discriminator complex stabilizes the discriminator in its 'stimulative' conformation. This stimulative complex bears enhanced affinity to the apo-receptor. Complexation with the aporeceptor (p.e. adenyl cyclase ${ }^{8}$ ) converts the apo-receptor to its functional state. In the case of the apo-receptor representing an enzyme, a substrate will be converted to a product which initiates one or more chains of metabolic events peculiar to the particular hormone and the particular target cell.

Actually, one hormone could react (in one cell type) with only one, or with a number of different discriminators. As a first approximation, we assume $\mathrm{ACTH}$ to react with only one type of discriminator in isolated fat cells (no experimental results opposed to this assumption). The hormone-discriminator complex, which is formed with one association constant (binding with the apo-receptor will, however, influence it to some unknown degree) then associates with one or more apo-receptors. In our case, adenyl cyclase and a 'lipolytic' receptor associated with 'transducer function' (Figure 5) will be influenced by the stimulative complex. The apo-receptors can bind the stimulative complex with different association constants, producing the effects of 


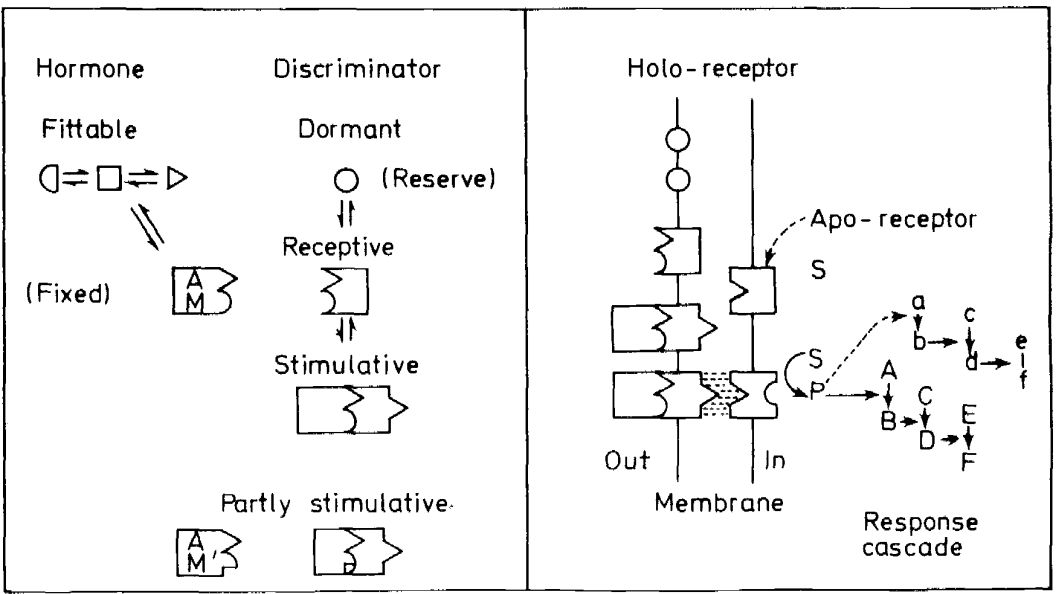

Figure 6. Hypothetical molecular receptor-model

'threshold' and 'receptor reserve' shifts reflected in $[\mathrm{H}]_{50}$. As de Haën ${ }^{8}$ has already pointed out, differences in maximal response can also easily be explained.

Hormone analogues with slightly altered 'message' would p.e. produce only partly stimulative discriminator complexes which would then bind with altered constants to the apo-receptors, producing shifts in absolute or relative potency and in maximum responses.

I wish to thank the Swiss Federal Institute of Technology and the Swiss National Foundation for Scientific Research for financial support of our work.

\section{REFERENCES}

1 R. Schwyzer, 'Peptides 1972 ', p. 424. Proceedings of the Twelfth European Peptide Symposium. North Holland: Amsterdam (1973).

2 E. W. Sutherland (Nobel Lecture), Angew. Chem. 84, 1117 (1972).

${ }^{3}$ G. A. Robison, R. W. Butcher and E. W. Sutherland, 'Cyclic AMP'. Academic Press: New York (1971).

4 L. Birnbaumer and M. Rodbell, J. Biol. Chem. 244, 3477 (1969).

5 H.-P. Bär and O. Hechter, Proc. Nat. Acad. Sci., Wash. 63, 350 (1969).

${ }^{6}$ M. Rodbell, L. Birnbaumer and S. L. Pohl, J. Biol. Chem. 245, 718 (1970).

7 I. Schorr, P. Rathnam, B. B. Saxena and R. L. Ney, J. Biol. Chem. 246, 5806 (1971).

${ }^{8}$ C. de Haën, personal communication (1973).

9 O. Hechter and Th. Braun, 'Structure Activity Relationships of Protein and Polypeptide Hormones', p 212. Excerpta Medica International Congress Series 241 : Amsterdam (1971).

${ }^{10}$ M. Rodbell, 'Hormones, Receptors, and Adenyl Cyclase Activity in Mammalian Cells'. p 59. Fogarty International Center Proceedings No. 4. National Institutes of Health: Bethesda, Md, USA (1969).

11 Ursula Lang and R. Schwyzer, FEBS Letters, 21, 91 (1972).

12 R.-J. Ho and E. W. Sutherland, J. Biol. Chem. 246, 6822 (1971).

13 P. Bally and K. Tilbury, 'Structure-Activity Relationships of Protein and Polypeptide Hormones', p 154. Excerpta Medica International Congress Series 161: Amsterdam (1968). 


\section{R. SCHWYZER}

14

1

Bar and O. Hechter, Biochem. Biophys. Res. Commun. 35, 681 (1969).

6 T. Braun and O. Hechter, 'Adipose Tissue', p 11. Georg Thieme: Stuttgart (1970).

17 R. Schwyzer, G. Karlaganis, R. Vogel and Ursula Lang, IRCS (Intern. Res. Commun. System) 73-3, 2- 2-1 (1973).

18 R. Schwyzer and G. Karlaganis, Liebig's Ann. Chem. 1298 (1973).

19 Ursula Lang, G. Karlaganis, S. Seelig, G. Sayers and R. Schwyzer, Helv. Chim. Acta, 56, 1069 (1973).

20 M. Rodbell, J. Biol. Chem. 239, 375 (1964).

21 P. Cuatrecasas, Proc. Nat. Acad. Sci., Wash. 68, 1264 (1971).

22 H.-U. Fisch, V. Pliška and R. Schwyzer, Europ. J. Biochem. 30, 1 (1972).

23 R. J. Lefkowitz, J. Roth and I. Pastan, Science, 170, 633 (1970).

24 R. J. Lefkowitz, J. Roth, W. Pricer and I. Pastan, Proc. Nat. Acad. Sci., Wash. 65. 745 (1970).

25 R. J. Lefkowitz, J. Roth and I. Pastan, Ann. NY Acad. Sci. 185, 195 (1971).

26 G. Sayers, S. Seelig, R. Schwyzer and P. Schiller, FEBS Letters, 19, 232 (1971).

27 R. J. Beall and G. Sayers, Arch. Biochem. Biophys. 148, 70 (1972).

${ }^{28}$ G. Sayers, S. Scelig, S. Kumar, G. Karlaganis, R. Schwyzer and M. Fujino, unpublished (1973)

29 J. Rudinger, V. Pliška and I. Krejči, Rec. Progr. Hormone Res. 28, 131 (1972).

${ }^{30}$ R. Schwyzer and P. Schiller, Hormones, Receptors, and Adenyl Cyclase Activity in Mammalian Cells, p 105. Fogarty International Center Proceedings No. 4. National Institutes of Health: Bethesda, Md, USA (1969).

31 R. Schwyzer and P. Schiller, Helv. Chim. Acta, 54, 897 (1971).

32 R. Schwyzer and P. Schiller, 'Peptides 1971', Proceedings of the Eleventh European Peptide Symposium, p 354. North Holland: Amsterdam (1973).

33 P. W. Schiller, Proc. Nat. Acad. Sci., Wash. 69, 975 (1972).

34 W. R. Moyle, Y. C. Kong and J. Ramachandran, J. Biol. Chem. 248, 2409 (1973).

35 M. Rodbell, J. Biol. Chem. 242, 5744 (1967).

${ }^{36}$ R. Schwyzer, Proceedings of the Fourth International Congress on Pharmacology (Schwabe \& Co.), 5, 196 (1969).

37 R. Schwyzer, unpublished results. 